\title{
Phenomena of sudden changes of water temperature in the coastal waters: comparison of upwelling events of Lake Baikal and Japanese lakes and coastal oceans
}

\author{
Tanaka Y. ${ }^{1 *}$, Katano T. ${ }^{1}$, Sugiyama M. ${ }^{2}$, Watanabe Y. ${ }^{3}$, Timoshkin O.A. ${ }^{4}$, \\ Troitskaya E.S. ${ }^{4}$, Shimaraev M.N. ${ }^{4}$, Drucker V.V. ${ }^{4}$ \\ ${ }^{1}$ Department of Ocean Sciences, TUMSAT, Tokyo, Japan \\ ${ }^{2}$ Department of Natural Resources, Kyoto University, Kyoto, Japan \\ ${ }^{3}$ Faculty of Science, Tokyo Metropolitan University, Tokyo, Japan \\ ${ }^{4}$ Limnological Institute, SB RAS, Irkutsk, Russia
}

\begin{abstract}
It has been known that, during the thermally stratified period, the water temperature in the coastal areas of Lake Baikal sometimes drops drastically, by several to ten degrees within only a few hours, and stay cold for a few days or more. In the recent quarter centuries, intensive observations have revealed such significant phenomena are related to the "coastal upwelling event" that is not rare, but rather common (seen several times every summer) in this great lake (Tanaka et al., 2005; Troitskaya et al., 2015). As similar phenomena exist in some other waters in the world, we here show a few examples from Japanese lakes and coastal waters.
\end{abstract}

Keywords: Lake Baikal, Lake Biwa, Pacific Ocean, Honshu Japan, coastal upwelling

In Lake Biwa, rapid decreases in the water temperature during the stratified period in the coastal waters occur (Kanari, 1970). They are related to the meteorological forcing due to storms (such as typhoons) and subsequent propagation of long internal waves. Also, in waters of the coastal ocean, similar phenomena have been recognized. For example, on the coast of the Kii Peninsula (facing the Pacific Ocean at the middle part of Honshu Island), long-term observations have revealed that rapid drops of water temperature occur in summer (Tanaka et al., 1992). These are due to the coastal upwelling being related to the passage of low pressures (including typhoons), and the weather front positioning. When the wind keeps blowing for several hours or more along a straight coastline (extending for several tens of kilometers or more), the water temperature responds to drop rapidly. These observations now made it realistic to predict the occurrence of the upwelling phenomena by obtaining an appropriate forecast of the local weather for several days or more.

In Lake Baikal, by systematically obtaining the temperature of the coastal waters together with the climatic properties, especially of the wind force and direction, and solar radiation, the mechanisms of the coastal upwelling could be made more precise, and the sudden changes in the water temperature during the stratified period could be statistically predicted.

We can imagine these phenomena affect the lives in the coastal waters. For example, when the cold water from a certain depth is lifted to the shallow, rich nutrients in the coastal waters must be fertilized even in the summer stratified period. Simultaneously, organisms living in the coastal zones should be eurythermal and exceptionally tolerant of such rapid changes in temperature; otherwise, some may not survive such a cold shock.

To understand the mechanisms, and to predict the occurrence of such phenomena should be of use in considering the conservation of the coastal environment. Systematized quantitative monitoring of the water environment and the climatic properties should be essential for the future understanding of Baikal's nature. Based on the actual and appropriate observations, bigdata analyses would help us understand nature's mechanisms.

\section{Acknowledgements}

This study has been supported by Grants-in-Aid for Scientific Research from the Ministry of Education, Culture, Sports, Science and Technology (MEXT), Japan (No. 18255001). 


\section{References}

Kanari S. 1970. Internal waves in Lake Biwa (I) The responses of the thermocline to the wind action. Bulletin of Disaster Prevention Research Institute 19(3): 19-26.

Tanaka Y., Katano T., Nakano S. et al. 2005. Violent fluctuations in water temperature along the western coast of Lake Baikal during the summer stratified period. In: The 4th Vereschagin Baikal Conference, p. 168.
Tanaka Y., Shinohara J., Tsuda R. 1992. The Kyucho in Uragami Bay. Bulletin on Coastal Oceanography 30: 37-44. (in Japanese)

Troitskaya E.S., Blinov V.V., Ivanov V.G. et al. 2015. Cyclonic circulation and upwelling in Lake Baikal. Aquatic Sciences 77: 171-182. DOI: 10.1007/s00027-014-0361-8 